\title{
A well differentiated neuroendocrine tumor of the jejunum with peritoneal carcinomatosis: A case report
}

\author{
FOTEINI ANTONIADOU ${ }^{1}$, DIMITRIOS KORKOLIS ${ }^{2}$, NEKTARIOS KOUFOPOULOS ${ }^{1}$, \\ DIMITRIOS MANATAKIS ${ }^{2}$ and STRATIGOULA SAKELLARIOU ${ }^{3}$
}

\author{
Departments of ${ }^{1}$ Pathology and ${ }^{2}$ Surgery, Saint Savvas Cancer Hospital of Athens, 11522 Athens; ${ }^{3}$ First Department \\ of Pathology, Medical School, National and Kapodistrian University of Athens, 11527 Athens, Greece
}

Received February 6, 2018; Accepted July 30, 2018

DOI: $10.3892 / \mathrm{mco} .2018 .1734$

\begin{abstract}
Neuroendocrine tumors (NETs), belong to a group of neoplasms that arise from neuroendocrine cells and express markers such as synaptophysin and chromogranin A. The digestive system (DS) is the most common site of NET development. The World Health Organization classification divides NETs into low grade (G1-G2) tumors (NETs) and high grade carcinomas [neuroendocrine carcinomas (NECs)], based on mitotic index and histological criteria. NET prognosis depends on tumor stage and grade. Low grade G1 NETs are characterized by a low proliferative rate and an indolent clinical course with a 5-year survival rate ranging between $38 \%$ (pancreas) and $88 \%$ (rectum). The present study reports a case of a low grade (G1) multifocal jejunal tumor with histologically confirmed features of aggressiveness, namely peritoneal carcinomatosis, lymph node metastasis and vascular carcinomatous emboli. Prediction of clinical behavior and survival in such a case is challenging. Although multiplicity and nodal metastases is not unusual for low grade NETs in this part of the gastrointestinal tract, peritoneal carcinomatosis is an extremely rare finding. Surgeons and histopathologists should be familiar with such eventualities and tumor boards are required in order to conclude whether aggressive therapeutic interventions may have any impact on patients' long term survival.
\end{abstract}

Correspondence to: $\mathrm{Dr}$ Foteini Antoniadou, Department of Pathology, Saint Savvas Cancer Hospital of Athens, 171 Alexandras Avenue, 11522 Athens, Greece

E-mail: fenia.antoniadou@gmail.com

Abbreviations: AJCC, American Joint Committee on Cancer; CAP, College of American pathologists; CT, computed tomography; CTCs, circulating tumor cells; DS, digestive system; G, grade; HPF, high power field; GI, gastrointestinal; NEC, neuroendocrine carcinoma; NET, neuroendocrine tumor

Key words: NET, jejunal neuroendocrine tumors, NET classification, peritoneal carcinomatosis

\section{Introduction}

Neuroendocrine tumors (NETs) arise from neuroendocrine cells, located in different tissues throughout the body. Gastroenteropancreatic NETs derive from neuroendocrine cells that are disseminated throughout the gastrointestinal (GI) tract or form neuroendocrine islets within the exocrine pancreatic tissue. Given its length, the GI tract is the largest neuroendocrine organ, enclosing more neuroendocrine cells than any other part of the human body (1). This could explain the fact that Digestive system (DS) is the most common site of NETs development, followed by the bronchopulmonary tree. Distribution of DS NETs at each specific site/organ is as follows: esophagus $<1 \%$, stomach $7 \%$, small intestine $17 \%$, appendix $5 \%$, colon $5 \%$, rectum $15 \%$, pancreas $45 \%$, liver $1 \%$ (2).

Grade $(\mathrm{G})$, based on Ki67 proliferation index and mitotic count, has proven to be a powerful prognostic indicator (3-6). Low grade DS NETs, are considered in general of good prognosis with a survival rate ranging between $38 \%$ (pancreas) and $88 \%$ (rectum). Lymph nodes and liver are the most common sites of metastases (20-50 and 60\% respectively) (2), while, to the best of our knowledge, peritoneal carcinomatosis has been reported only once in G1 gastrointestinal NETs (7).

\section{Case report}

Written informed consent was obtained from the patient. A 60-year-old, female, Caucasian patient, with an unremarkable past medical history, was admitted in a provincial hospital, with a 20-day history of atypical, crampy abdominal pain, vomiting and diarrhea. Initial diagnostic work-up included an upper and lower gastrointestinal (GI) endoscopy, both of which were normal, and an abdominal CT scan, which revealed mild dilatation of jejunal loops. Patient's basic laboratory values and tumor markers (CEA, Ca19-9) were within normal range. She was discharged with a diagnosis of recurrent partial bowel obstruction and was referred to the GI Department of a tertiary hospital (Agios Savvas Anticancer Hospital, Athens, Greece), for further investigation.

Due to worsening clinical condition, patient was transferred to the Department of Surgical Oncology with a working diagnosis of complete small bowel obstruction. An exploratory 
laparotomy was performed, which revealed multiple tumoral lesions in the wall of the jejunum, causing complete intestinal obstruction. Due to extensive regional lymphadenopathy up to the root of the associated mesentery, an extensive resection of jejunum was performed, with primary anastomosis. Inspection of the abdominal cavity did not reveal hepatic or other splanchnic metastases. However, a suspicious peritoneal nodule measuring $2 \mathrm{~cm}$ in the pelvic cul-de-sac was excised for biopsy. Postoperative course was uneventful and patient was discharged on the 8th postoperative day.

The surgical specimen consisted of a 76-cm-long small intestine segment obstructed at a distance of $20 \mathrm{~cm}$ from the distal resection margin and dilated proximal to the obstruction. Obstruction was caused by 4 whitish neoplastic foci measuring $7,8,12$ and $20 \mathrm{~mm}$ in maximum diameter located in the jejunal wall causing mucosal erosion. Mesenteric fat seemed to be macroscopically involved by the neoplasm. Forty nine lymph nodes were retrieved measuring 2-10 mm, 2 of which from the mesentery root. Twenty five tissue cassettes were prepared including 1 from each neoplastic focus (4 cassettes), 1 from the peritoneal nodule, 1 from nonlesional mucosa, 2 from the intestinal resection margins and 17 containing the dissected lymph nodes. Tissue cassettes were processed according to standard protocol. H\&E sections were examined by 2 specialist pathologists (AF and SS) with interest in neuroendocrine tumors.

Sections showed variable sized solid nests composed of uniform neoplastic cells with bland nuclei and salt and pepper chromatin. Tumoral cells invaded widely the intestinal wall and perivisceral fat, abating but not penetrating the serosa (Fig. 1A and B). Mitotic activity assessed in 60 tumoral HPFs was very low resulting in a mitotic count $<2$ per $10 \mathrm{HPF}$ in the hot spots. Moreover, plenty of blood/lymph vessel emboli and foci of perineural infiltration were recognized (Fig. 1C). Of the 47 lymph nodes dissected from the perivisceral fat, 6 were positive for metastatic disease (Fig. 1D). The 2 additional lymph nodes excised from the root of the mesentery were also positive for disease. Finally, the nodule that was found in the pelvic cul-de-sac was proven a metastatic implant.

Tumor immunohistochemical analysis was performed in 3 tissue blocks (from the 2 largest neoplastic foci and the peritoneal nodule) using antibodies against chromogranin A, synaptophisin, serotonin and Ki67. The EnVision Staining System (Dako, Glostrup, Denmark) with 3,3' diaminobenzidine (DAB) as a chromogen, and a PT Link, Pre-Treatment Module was applied according to standard protocols. Table I shows details on antibodies and methodology. Diffuse positivity was evident for chromogranin A (Fig. 1E), synaptophysin and serotonin. The percentage of Ki67 positivity in 2,000 neoplastic cells was estimated in areas of strongest nuclear labeling by the 2 pathologists independently. Both of them concluded that Ki67 proliferation index was $<2 \%$ (Fig. 1F). Histological diagnosis was NET Grade1, pT3N1M1 (TNM 7th edition, 2007), stage IV, according to the 2010 American Joint Committee on Cancer (AJCC). The patient received m-TOR inhibitor (Everolimus) and is free of disease 18 months post surgery.

\section{Discussion}

Natural history of NETs is poorly understood. They arise from enterochromaffin cells, which are multipotent stem cells
Table I. Antibodies used for histological diagnosis.

\begin{tabular}{lc}
\hline Antibody type & $\begin{array}{c}\text { Antibody } \\
\text { dilution }\end{array}$ \\
\hline $\begin{array}{l}\text { Synaptophysin } \\
\text { (mouse monoclonal AB, clone DAK-SYNAP) }\end{array}$ & $1: 50$ \\
$\begin{array}{l}\text { Chromogranin A } \\
\text { (mouse monoclonal AB, clone DAK-A3) }\end{array}$ & $1: 100$ \\
$\begin{array}{l}\text { Serotonin (mouse monoclonal AB, } \\
\text { clone 5HT-H209) }\end{array}$ & $1: 50$ \\
\begin{tabular}{l} 
Ki67 (mouse monoclonal AB, clone MIB-1) \\
\hline
\end{tabular} & $1: 50$ \\
\hline
\end{tabular}

$\mathrm{AB}$, antibody. All antibodies were purchased from Dako; Agilent Technologies, Inc. and incubated for $45 \mathrm{~min}$ at room temperature. Method of antigen retrieval: PT Link, Pre-Treatment Module.

Table II. Grading system of gastrointestinal neuroendocrine neoplasms (World Health Organization 2010).

\begin{tabular}{lcc}
\hline Grade & Mitotic count $(/ 10 \mathrm{HPF})$ & Ki-67 index $(\%)$ \\
\hline G1 & $<2$ & $<2$ \\
G2 & $2-20$ & $3-20$ \\
G3 & $>20$ & $>20$ \\
\hline
\end{tabular}

G, grade; HPF, high powered field.

that migrate from the neural crest to the gut ectoderm (8-10). According to the embryological origin, NETs are classified as foregut, midgut or hindgut. Foregut NETs refer to tumors arising in the respiratory tract, thymus, thyroid, stomach, duodenum and pancreas. Midgut NETs develop in the small bowel, appendix and ascending colon (11), while hindgut tumors appear in the transverse, descending colon and rectum $(12,13)$.

NETs can be functional $(40 \%)$ or non functional $(60 \%)$ depending on the excess of hormones (serotonin, substance P) and/or peptides (chromogranin, synaptophysin) secretion. Functional NETs can cause symptoms such as flushing (95\%), secretory diarrhea (78\%) and abdominal crumps (50\%) (14-16). Non functional NETs can grow undetected for years, causing symptoms in later stages due to mass effect, such as intestinal blockage or bleeding. The majority of NETs are sporadic and only $10 \%$ are familial, arising in the context of autosomal dominant inherited syndromes (MEN1-2, VHL, NF) $(17,18)$.

NET's are the second most prevalent group of tumors in the GI tract. Through the years, WHO has applied various classifications to GI NETs. In 1980 GI NETs were categorized as follows: I. Carcinoid, II. Mucocarcinoid, III. Mixed forms (Carcinoid-Adenocarcinoma) and IV. Pseudotumor lesions. In 2000, WHO revised the previous classification in the following categories: I. Well differentiated endocrine carcinoma (WDEC), II. Poorly differentiated/small cell carcinoma (PDEC), III. Mixed endocrine-exocrine carcinoma (MEEC) and III. Tumor - like lesions.

Over the years, it was shown that proliferation rate, described as the number of mitoses per $10 \mathrm{HPF}$ and the percentage of 

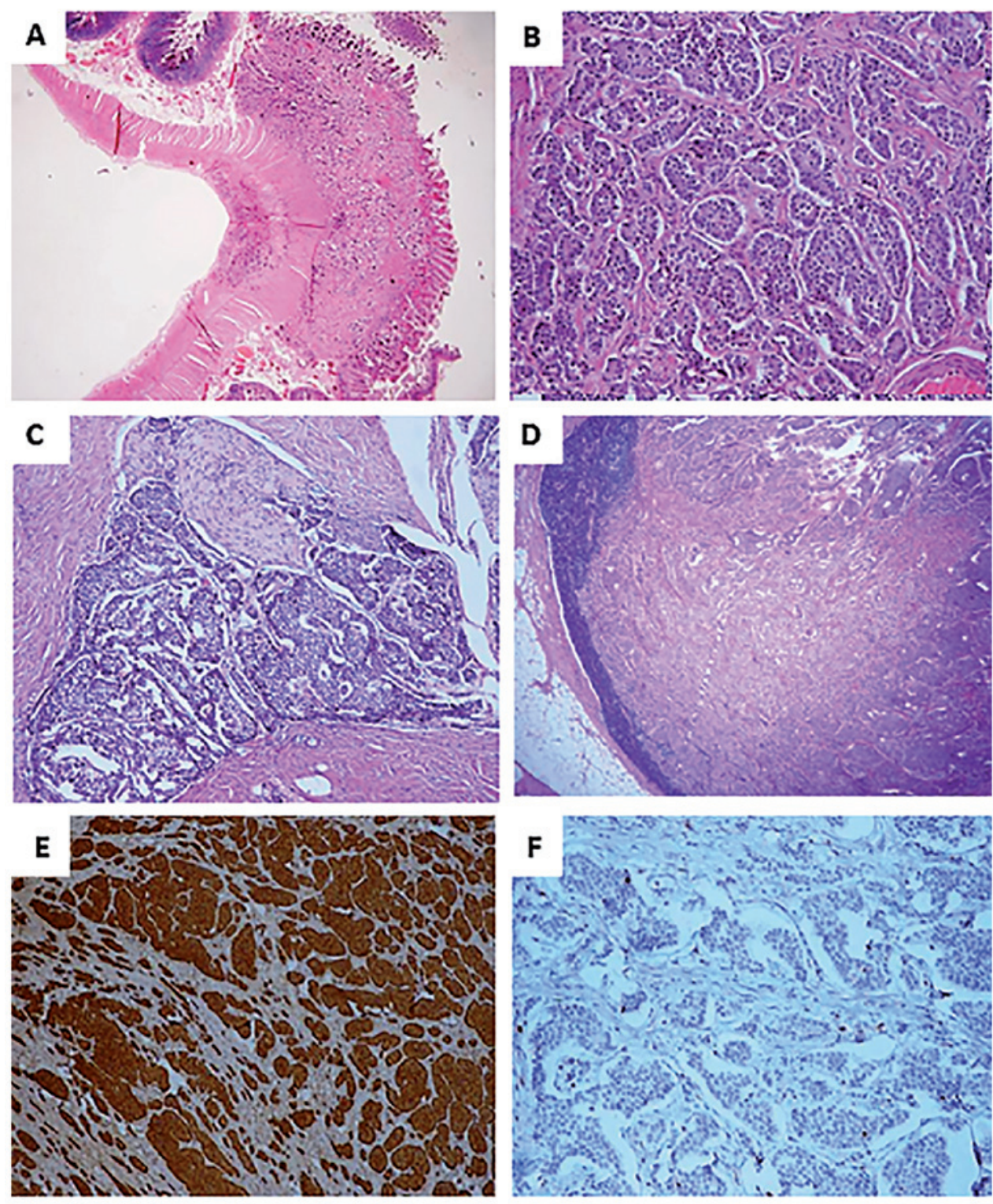

Figure 1. Tumor histological features. (A) Jejunal wall infiltrated by the tumor (H\&E; magnification, x10). (B) Tumor composed of uniform neoplastic cells arranged in solid nests (HE; magnification, x200). (C) Tumor perineural infiltration (H\&E; magnification, x100). (D) Lymph node metastasis (H\&E; magnification, x40). (E) Diffuse tumor positivity for synaptophysin (synaptophysin immunostaining, DAB chromogen; magnification, x100). (F) Ki67 tumor proliferation index (Ki67/MIB1 immunostaining, DAB chromogen; magnification, x100). H\&E, hematoxylin and eosin; DAB, 3,3'-diaminobenzidine.

Ki67 positive neoplastic cells, provides significant prognostic information for NETs, such as recurrence or metastatic potential (3). In 2010 a new classification was established, grading GI NETs based on mitotic count and Ki67 index (percentage of Ki67 positive cells in 500-2,000 cells counted in areas of strongest nuclear label), as follows: I. NET Grade 1: Ki67 1-2\% and/or up to 1 mitosis/10 HPF, II. NET Grade 2: Ki67 3-20\% and/or 2-20 mitoses/10 HPF, II. Neuroendocrine carcinomas (NECs): Ki67 $>20 \%$ and/or $>20$ mitoses/10 HPF. III. Mixed adeno-neuroendocrine carcinoma (MANEC): $\geq 30 \%$ of tumor cells with neuroendocrine phenotype. Tables II and III, show the latest grading GI NETs classification and Table IV presents the transition from previous classifications to the new grading categories (4).

Difficulties arise when applying NET WHO 2010 classification in practice. First of all, categorization of NETs with a Ki67 index between 2 and 3\% is unclear. To address this
Table III. World Health Organization 2010 classification of gastrointestinal neuroendocrine neoplasms.

Type of neuroendocrine neoplasm

Description

NET

Low to intermediate grade (G1-G2), well to moderately differentiated neoplasms

NEC (small cell to High grade (G3), moderate to poorly large cell type) differentiated neoplasms

MANEC

Neoplasms with $\geq 30 \%$ of tumor cells that have NE characteristics

NET, neuroendocrine tumors; NEC, neuroendocrine carcinoma; MANEC, mixed adeno-neuroendocrine carcinoma; G, grade. 
Table IV. WHO classifications of gastrointestinal neuroendocrine neoplasms.

\begin{tabular}{|c|c|c|c|}
\hline Classification no. & WHO (1980) & WHO (2000) & WHO (2010) \\
\hline 1 & $\begin{array}{l}\text { Carcinoid islet } \\
\text { cell tumor }\end{array}$ & $\begin{array}{l}\text { Well differentiated } \\
\text { endocrine tumor }\end{array}$ & NET G1 \\
\hline 2 & Muco-carcinoid & $\begin{array}{l}\text { Well differentiated } \\
\text { endocrine carcinoma }\end{array}$ & NET G2 \\
\hline 3 & $\begin{array}{c}\text { Mixed forms } \\
\text { carcinoid -adenocarcinoma }\end{array}$ & $\begin{array}{l}\text { Poorly differentiated } \\
\text { endocrine carcinoma }\end{array}$ & Neuroendocrine carcinoma \\
\hline 4 & Pseudotumor lesions & $\begin{array}{l}\text { Mixed endocrine-exocrine } \\
\text { cell neoplasm }\end{array}$ & $\begin{array}{l}\text { Mixed } \\
\text { adeno-endocrine carcinoma }\end{array}$ \\
\hline 5 & - & Tumor-like lesions & - \\
\hline
\end{tabular}

WHO, World Health Organization; NET, neuroendocrine tumors; G, grade.

issue, Yamaguchi et al (5) studied retrospectively 45 NET G1/G2 cases and showed that the cutoff value for predicting metastases or recurrence was $2.8 \%$. They concluded that the categorization of NETs into G1 or G2 based on Ki67 index of $3 \%$ can predict metastases or recurrences (5).

Apart from grade, stage, referring to tumor size, extent of invasion and metastatic status is an indispensable tool for therapeutic intervention and prognosis estimation and should always be taken into consideration (19). According to some epidemiological data from a 6 year surveillance study in USA during the period of 1988-2004, medium survival was 203 months for localized tumors, 114 months for tumors with regional extension and 39 months for distant metastatic tumors (20). The TNM classification of Malignant Tumours, the most widely used organ/site specific cancer staging system, is also applied to GI NETs. The recently published 8th edition of TNM classification acknowledges the importance of the number of lymph nodes metastasis and the presence of mesenteric mass, incorporating for the first time this information in the $\mathrm{N}$ category of the TNM system (21). According to the new classification, presence of mesenteric neoplastic mass measuring more than $2 \mathrm{~cm}$ in maximum diameter corresponds to $\mathrm{N} 2$ category, even in the absence of lymph node metastasis. Another novelty is that the new M1 category (distant metastasis) includes 3 sub-categories, namely hepatic metastasis only (M1a), extrahepatic metastasis only (M1b) and hepatic and extrahepatic metastase (M1c). Concerning the presented case, the neoplastic mass found adhered to the peritoneum of the rectouterine pouch (cul-de-sac) does not qualify for a mesenteric mass. On the other hand and despite the improvements in the new TNM classification, it remains unclear whether it should be considered an extrahepatic metastasis.

Recent data suggest that useful information concerning NET clinical outcome could be derived from circulating tumor cells (CTCs) expressing epithelial cell adhesion molecule (EpCAM), possibly with more predictive power than WHO grading system (22). However, CTCs as prognostic biomarkers cannot be widely used at present time.

The therapeutic options for NETs are the following: i) Surgery: Curative (rarely), ablative (very often); ii) debulking: Radiofrequency ablation (RFA)/embolization, chemoembolization/radioembolization; irradiation, external (bone, brain metastasis)/tumor targeted, radioactive therapy; iii) medical therapy: Chemotherapy, biological treatment (somatostatin analogs, a-Interferon, m-TOR inhibitors, VEGF R inhibitors, Other TKI's (23).

The presented case is of special interest regarding its prognosticators. On one hand, tumor grade, according to proliferation rate, is low (G1), suggesting an indolent clinical course. On the other hand, many histological features, namely, multiplicity and size of tumor, depth of invasion, lymphatic and vascular emboli, nodal metastases and peritoneal implant, point towards aggressive tumor behavior. According to English literature, small intestinal NETs have a tendency to multiplicity (30\%) and those multiple tumors have been associated with a worse clinical outcome $(24,25)$. Microscopic tumors, as small as $3 \mathrm{~mm}$, can give rise to nodal and distant metastases. The current protocol of the College of American Pathologists (CAP), cites a $12 \%$ frequency of lymph node metastasis for small intestinal low grade NETs measuring $<1 \mathrm{~cm}$ (13). It is not clear why low grade tumors in this location present with aggressive histological features and it seems possible that their behavior is underestimated.

As far as peritoneal carcinomatosis is concerned, it represents a complication encountered in high grade tumors (7). To the best of our knowledge, only one additional to the present case NET G1, Stage IV with peritoneal carcinomatosis has been previously reported (7). Prognosis of GI NETs depends both on stage and grade. The 5-year survival rate for stages I-III is $70-80 \%$ and for stage IV, $35-80 \%$. Patients with G1 NETs have a $94 \%$ 5-year survival, with G2, $83 \%$ and with G3, 50\% (26). Prognosis of a well differentiated NET with peritoneal carcinomatosis cannot be estimated, since neither the WHO 2010 classification nor the TNM system, even in the recently published 8th edition, can be useful for prognostic stratification. On occasion of the present case, it becomes evident that criteria for metastatic disease should be reconsidered to include peritoneal metastases, in order to provide patients with the most suitable therapeutic scheme (27).

We reported a case of a jejunal G1, stage IV NET, with peritoneal carcinomatosis. Although multiplicity and nodal metastases is not unusual for low grade NETs in this part of the GI tract, peritoneal carcinomatosis is an extremely rare finding. Surgeons and histopathologists should be familiar 
with such eventualities and tumor boards need to conclude whether aggressive therapeutic interventions may have any impact on patients' long term survival.

\section{Acknowledgements}

Not applicable.

\section{Funding}

No funding was received.

\section{Availability of data and materials}

All data generated or analyzed during this study are included in this published article.

\section{Authors' contributions}

FA conceived the study and drafted the manuscript. DK interpreted the data and revised the manuscript critically for important intellectual content. NK designed the study, acquired the data and drafted the manuscript. DM analyzed the data and revised the manuscript. SS interpreted the data and revised the manuscript critically for important intellectual content. All authors approved the final version of the manuscript for publication and have agreed to be accountable for all aspects of the work.

\section{Ethics approval and consent to participate}

Written informed consent was obtained from the patient.

\section{Patient consent for publication}

Written informed consent was obtained from the patient.

\section{Competing interests}

The authors declare that they have no competing interests.

\section{References}

1. Rehfeld JF: The new biology of gastrointestinal hormones. Physiol Rev 78:1087-108, 1998.

2. Hauso O, Gustafsson B, Kidd M, Waldum H, Drozdov I, Chan A and Modlin I: Neuroendocrine tumour epidemiology. Cancer 113: 2655-2664, 2008.

3. Rindi G, Petrone G and Inzani F: The 2010 WHO classification of digestive neuroendocrine neoplasms: a critical appraisal four years after its introduction. Endocr Pathol 25: 186-192, 2014.

4. Rindi G, Arnoid R, Bosman FT, Capella C, Klimstra DS, Kloppel G, Komminorth P and Solcia E. Nomeclature and classification of the neuroendocrine neoplasms of the digestive system. In: WHO Classification of Tumours of the Digestive System. 4th edition. IARC Press, Lyon, France, pp13-14, 2010.

5. Yamaguchi T, Fujimori T, Tomita S, Ichikawa K, Mitomi H, Ohno K, Shida Y and Kato H: Clinical validation of the gastrointestinal NET grading system: Ki67 index criteria of the WHO 2010 classification is appropriate to predict metastasis or recurrence. Diagn Pathol 8:65, 2013.

6. Karakus E, Helvacı A, Ekinci O, and Dursun A: Comparison of WHO 2000 and WHO 2010 classifications of gastroenteropancreatic neuroendocrine tumours. Turk J Gastroenterol 25: 81-87, 2014.
7. Celotti A, Pulcini G, Schieppati M, Ministrini S, Berruti A, and Ronconi M: An unususal case of a well-differentiated neuroendocrine tumor of the ileum with peritoneal carcinomatosis : A case report.World J Surg Oncol 13: 1353-1361, 2015.

8. Langley K: The neuroendocrine consept today. Ann NY Acad Sci 733: 1-17, 1994.

9. Warner RR: Enteroendocrine tumors other than carcinoid. A review of clinically significant advances. Gastroenterology 128: 1668-1684, 2005

10. Uberg K: Carcinoid tumors: Current concepts in diagnosis and treatment. The Oncologist 3: 339-45, 1998.

11. Griniatsos J and Michail O: Appendiceal neuroendocrine tumors. Resent insights and clinical implications. World J Gastrointest Oncol 2: 192-96, 2010.

12. Ni S, Sheng W, Du X and Sheng W: Pathologic research update of colorectal neuroendocrine tumors. World J Gastroenterol 16: 1713-19, 2010.

13. Konishi T, Watahabe T, Nagawa H, Oya H, Ueno M, Kuroyanagi H, Fujimoto Y, Akiyoshi T, Yamaguchi T and Muto T: Treatment of colorectal carcinoids. A new paradigm. World J Gastrointest Surg 2: 153-56, 2010.

14. Begum N, Maasberg S, Plockinger U, Anlauf M, Rinke A, Popperl G, Lehnert H, Izbicki HR, Krausch M, Vashist YK et al: Zentralbl Chir 139: 276-283, 2014.

15. Modlin I.M, Shapiro MD and Kidd M: Signified Oberdorfer. Origins and perspectives of carcinoid tumors. Human Pathology 35: 1440-51, 2004.

16. Krols LK: Carcinoid tumors and the carcinoid syndrome. What is new in therapeutic pipeline. The carcinoid cancer foundation: carcinoid symposium 2002. http://www.carcinoid.org. Accessed July 8, 2017.

17. Oberg K: The genetics of neuroendocrine tumors. Semin Oncol 40: 37-44, 2013

18. Kaltsas G, Besser M and Grossman A: The diagnosis and medical management of advanced neuroendocrine tumors. Endocr Rev 25: 458-511, 2004.

19. Jamalli $\mathrm{M}$ and Chetty R: Predicting prognosis on gastroenteropancreatic neuroendocrine tumors. An overview and the value of Ki 67 immunostaining. Endocr Pathol 19: 282-288, 2008.

20. Yao JC, Hassan M, Phan A, Dagohoy C, Leary C, Mares JE, Abdalla EK, Fleming JB, Vauthey JN, Rashid A et al: One hundred years after 'carcinoid'. Epidemiology of and prognostic factors for neuroendocrine tumors in 38,525 cases in the United States. J Clin Oncol 26: 3063-3072, 2008.

21. Well-Differentiated Neuroendocrine Tumours of the Gastrointestinal Tract. In: Union for International Cancer Control (UICC). TNM Classification of Malignant Tumours. 8th edition. Wiley-Blackwell, Hoboken, NJ, pp 96-99, 2017.

22. Khan MS, Kirkwood A, Tsigani T, Garcia-Hernnandez I, Hartley JA, Caplin ME and Meyer T: Circulating tumor cells as prognostic markers in neuroendocrine tumors. J Clin Oncol 31: 365-372, 2013.

23. Mei D, Alexandria T and James C: New strategies for advanced neuroendocrine tumors. CCR 18: 1830- 37, 2012.

24. Burke AP, Thomas RM. Elsayed AM and Sobin H: Carcinoids of jejunum and ileum: Cancer 79: 1086-93, 1997.

25. Yantiss RK, Odze RD, Farraye FA and Rosenberg AE: Solitary versus multiple carcinoid tumors. A clinical and pathologic review of 68 cases. Am J Surg Pathol 27: 811-817, 2003.

26. Jann H, Roll S, Couverald A, Hentic O, Pavel M, Muller-Nordhorn J, Koch M, Rocken C, Rindi G, Ruszniewski P, et al: Neuroendocrine tumors of midgut- hindgut origin. Tumor-node-metastasis classification determines clinical outcome. Cancer 117: 3332-3341, 2011.

27. Shi Ch, Adsay V, Bergsland EM, Berlin J, Branton PA, Fitzgibbons PL, Frankel WL, Kakar S, Klepeis V, Klimstra DS, et al: Protocol for the Examination of Specimens from Patients with Neuroendocrine Tumors (Carcinoid Tumors) of the Jejunum and Ileum. Version: Jejunum/Ileum NET 1.0.0.0. Cancer protocol templates. College of American Pathologists (CAP), 2017. http://www.cap.org/ShowProperty?nodePath=/UCMCon/Contribution $\% 20$ Folders/WebContent/pdf/cp-jejunal-Ileal-net-17protocol-1000.pdf. Accessed July 8, 2017. 